\title{
Combined strength and aerobic training increases transforming growth factor- 61 in patients with type 2 diabetes
}

\author{
Anna-Maria Touvra, ${ }^{1}$ Konstantinos A. Volaklis, ${ }^{1}$ Apostolos T. Spassis, ${ }^{1}$ Christos E. Zois, ${ }^{1}$ \\ Helen T. Douda, ${ }^{1}$ Kalliopi Kotsa, ${ }^{2}$ Savvas P. Tokmakidis ${ }^{1}$ \\ ${ }^{1}$ Department of Physical Education and Sport Sciences, Democritus University of Thrace, Greece, ${ }^{2}$ Department of \\ Endocrinology, Aristotle University of Thessaloniki, Thessaloniki, Greece
}

\begin{abstract}
OBJECTIVE: The aim of the present study was to examine the effects of a combined strength and aerobic training program on pro- and anti-inflammatory cytokines and transforming growth factor- $\beta 1$ in patients with type 2 diabetes. DESIGN: Ten patients with type 2 diabetes, aged 55.5 (5) years [median (IQR)] participated in a supervised systematic exercise training program which included aerobic exercise and strength training, undertaken four days per week for eight weeks. RESULTS: The training program increased transforming growth factor- $\beta 1$ concentration $(+50.4 \%)$ and reduced high sensitivity $C$ reactive protein levels $(-24.1 \%)$ without altering the levels of interleukin-6, interleukin-10, interferon- $\gamma$ and tumor necrosis factor- $\alpha$. Additional improvements were also achieved in anthropometric characteristics, glycated hemoglobin $\left(\mathrm{HbA}_{1 \mathrm{c}}: \mathbf{- 1 1 . 8 \%}\right.$ ), homeostasis model assessment of insulin resistance index (HOMA-IR: -15\%) and physical fitness parameters (stress test: $+\mathbf{2 6 . 6 \%}$, upper muscle strength: $\mathbf{+ 3 2 . 4 \%}$ and lower muscle strength: $+48.9 \%$ ). CONCLUSION: A combined strength and aerobic exercise program has a potential anti-atherogenic and anti-inflammatory impact which most likely reduces the risk of cardiovascular disease and improves the health status in patients with type 2 diabetes.

Key words: Cytokines, Exercise, Training effects, Transforming growth factor- $\beta 1$, Type 2 diabetes
\end{abstract}

\section{INTRODUCTION}

There is accumulating evidence that a subclinical inflammatory reaction precedes the onset and

Address for correspondence:

Savvas P Tokmakidis, Department of Physical Education and Sport Science, Democritus University of Thrace, Komotini 69100, Greece, Tel.: +30 25310 39649, Fax: +30 2531039724 , e-mail: stokmaki@phyed.duth.gr

Received 05-11-10, Revised 10-02-11, Accepted 30-02-11 the progression of type 2 diabetes (DM2). ${ }^{1}$ Diabetic patients suffer from atherosclerosis, which involves both inflammatory and immunological mechanisms. ${ }^{2,3}$ Chronic low grade inflammation is reflected in raised C reactive protein (CRP) concentrations, an independent predictor of cardiovascular disease, ${ }^{4}$ as well as in elevated serum levels of pro-inflammatory cytokines. ${ }^{5}$

Transforming growth factor- $\beta 1$ (TGF- $\beta 1$ ), a multifunctional protein, acts as an anti-inflammatory 
cytokine $^{6}$ and restrains atherosclerosis development. ${ }^{7}$ Specifically, TGF- $\beta 1$ has an immunoregulatory role and functions as an agent that protects against the initial formation of fatty streak lesions and balances the environment of the vessels. ${ }^{8}$ By contrast, the inhibition of TGF- $\beta 1$ signalling has negative effects which allow the development of atherosclerotic lesions. ${ }^{9}$ In addition, increased serum levels of TGF- $\beta 1$ are associated with extended survival and reduced incidence of coronary events in patients with coronary artery disease, ${ }^{10}$ which constitutes the main cause of morbidity and mortality in patients with DM2. Therefore, it is important to study the TGF- $\beta 1$ activity in patients with DM2.

The expression of TGF- $\beta 1$ increases in response to mechanical stimuli in vascular tissue, ${ }^{11}$ while regular exercise suitably activates the vascular bed. Thus, apart from the beneficial effects on glucose control in DM2, ${ }^{12}$ exercise can contribute to the prevention of atherosclerosis by modifying several related pathogenetic factors. ${ }^{13,14}$

Recent guidelines provide strong evidence for the beneficial effect of exercise training, either aerobic or resistance training, in patients with DM2. ${ }^{15}$ Combined strength and aerobic exercise training has been established as the most effective mode in terms of glucose control, insulin action and the modification of cardiovascular risk factors. ${ }^{16-21}$ Recently, the antiinflammatory effects of exercise training have also been documented. ${ }^{22}$ However, studies concerning the effects of exercise on inflammatory markers in patients with DM2 ${ }^{23-25}$ are not consistent and more data are required. Furthermore, there is no evidence as to how exercise affects the levels of serum TGF- $\beta 1$ in patients with DM2. Previous studies have demonstrated that resistance exercise is effective in increasing serum levels of TGF- $\beta 1$ in healthy adults, ${ }^{26}$ and Gordon et $\mathrm{al}^{27}$ showed that resistance exercise increases TGF- $\beta 1$ transcription in skeletal muscle of patients with DM2.

The aim of the present study was to examine the effects of combined strength and aerobic training on glycemic control and inflammatory status in patients with DM2. We assumed that two months of training would increase TGF- $\beta 1$ and other anti-inflammatory markers and reduce pro-inflammatory markers.

\section{METHODS}

\section{Subjects}

Ten patients, (eight women - postmenopausal for at least 10 years - and two men), non-smokers, aged 55.5 (5) years, with DM2 for 6.5 (6) years and glycated hemoglobin $\left(\mathrm{HbA}_{1 \mathrm{c}}\right)$ levels at $7.2(1.5) \%$, participated voluntarily in a supervised systematic exercise training program. Values represent median (IQR). Seven patients were treated with monotherapy (metformin) and three were treated with a combination (metformin and sulfonylurea or glitazone). There was no alteration in the prescribed medication two months before and throughout the study. The study was approved by the institutional ethics committee.

\section{Experimental design}

The exercise training programme involved combined aerobic and strength training and was performed four days per week for eigh weeks. Every session included a warm-up period (10 minutes), the main programme (60 minutes) and a cool-down period (10 minutes). The main programme included 30 minutes of aerobic exercise at $70-80 \%$ of the maximal heart rate achieved during a symptom-limited graded exercise, followed by the strength training. Strength training consisted of 3 sets of 15 repetitions on leg press, knee extension, abductors, bench press, pec-deck, rowing. The weight load for each exercise was set at $60 \%$ of the maximum weight that could be lifted in one repetition (1RM) with 1 minute rest between sets and 2 minutes rest between exercises.

\section{Measurements}

Height and body weight were measured and body mass index (BMI) was calculated. Waist circumference measurement was recorded as the mean of two measurements, the first one on the navel point and the second $3 \mathrm{~cm}$ above the first point. Blood samples were obtained under the same conditions after an overnight fast, at baseline and 3 days after the exercise training program. Each participant completed a 72hour dietary record before the baseline measurement, which was followed with consistency 72 hours prior to the final measurement. Plasma was separated 30 minutes after centrifugation at $3000 \mathrm{~g}$ at $4^{\circ} \mathrm{C}$ and stored at $-80^{\circ} \mathrm{C}$ until further analysis. Insulin resistance was calculated using the homeostasis model assess- 
ment (HOMA-IR) by the following formula: fasting plasma glucose $(\mathrm{mmol} / \mathrm{L}) \mathrm{x}$ fasting serum insulin $(\mu \mathrm{U} /$ $\mathrm{mL}) / 22.5 .^{28}$ Serum cytokines were measured using a commercially available ELISA kit based on the immunoenzymatic method. In particular, TGF- $\beta 1$ and high sensitivity CRP (hs)-CRP were measured by kits of DRG International, Inc., while interleukin (IL)-6 and IL-10, interferon- $\gamma($ INF- $\gamma)$ and tumor necrosis factor- $\alpha$ (TNF- $\alpha)$ by kits of eBioscience Inc.

Maximum total muscle strength was measured with the one-repetition maximum (1-RM) method and was calculated as the sum of two exercises, leg extension and bench press. A rest of $1 \mathrm{~min}$ followed each trial and the resistance was increased by approximately 2.5 or $5 \mathrm{~kg}$ when the patient was near to maximum. Patients underwent a symptom-limited graded exercise test protocol on a treadmill using the Bruce protocol. Blood pressure was measured manually at rest and during the stress test. Heart rate was measured using a 12-lead electrocardiograph with an automatic ST-segment analysis. These measurements were conducted by the same investigator.

\section{Statistical analysis}

Data analyses were carried out using the Statistical Package for the Social Sciences (version 12.0.1; SPSS, Chicago, IL). Results are presented in text, table and figures as median and the interquartile range (IQR). The baseline (pre) values were compared with the endpoint (post training) values within groups by paired samples using the Wilcoxon test. The $\mathrm{p}$ value $<0.05$ was specified as a statistically significant finding.

\section{RESULTS}

The exercise training program applied in this study significantly increased TGF- $\beta 1$ serum concentration by $50.4 \%(\mathrm{p}=0.012$; Figure 1$)$ and reduced hs-CRP levels by $24.1 \%(p=0.017$; Figure 2$)$. No significant alterations were found in the concentrations of IL6 , IL-10, INF- $\gamma$ and TNF- $\alpha$ throughout the study (Table 1). Considerable improvement was achieved in anthropometric characteristics (BMI: $p=0.007$, waist circumference: $p=0.005)$ and glucose regulation with $11.8 \%$ reduction in $\mathrm{HbA}_{1 \mathrm{c}}(\mathrm{p}=0.016)$ but no significant change in HOMA-IR $(\mathrm{p}=0.508)$. Physical fitness parameters increased significantly $(\mathrm{p}<0.05$; Table 1). Stress test duration increased from 7.2 (2.5)

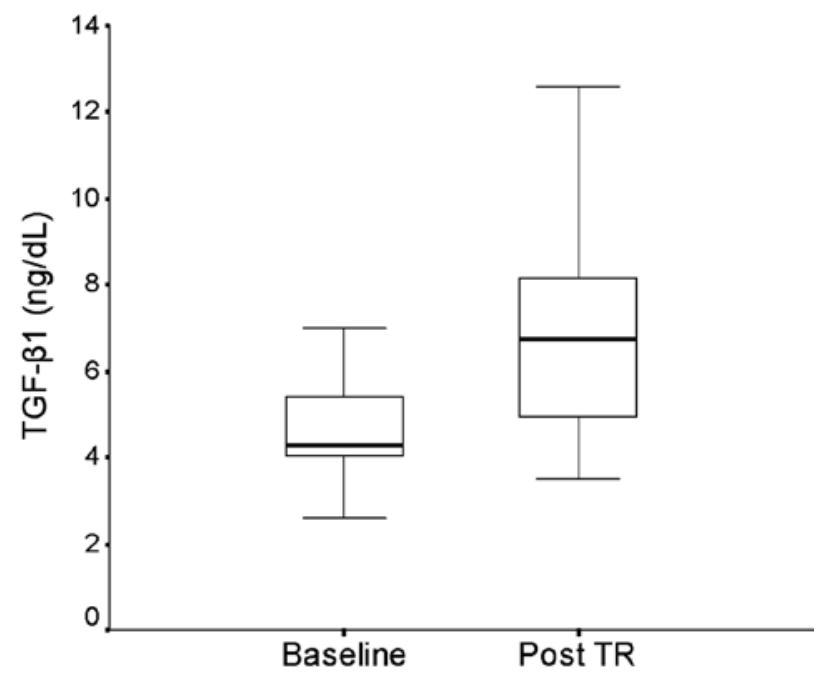

Figure 1. The effects of a combined strength and aerobic exercise training program (Post TR) on transforming growth factor $-\beta 1$ (TGF- $\beta 1 ; p=0.012)$. Values are presented as median and the length of the boxes represents the interquartile range.

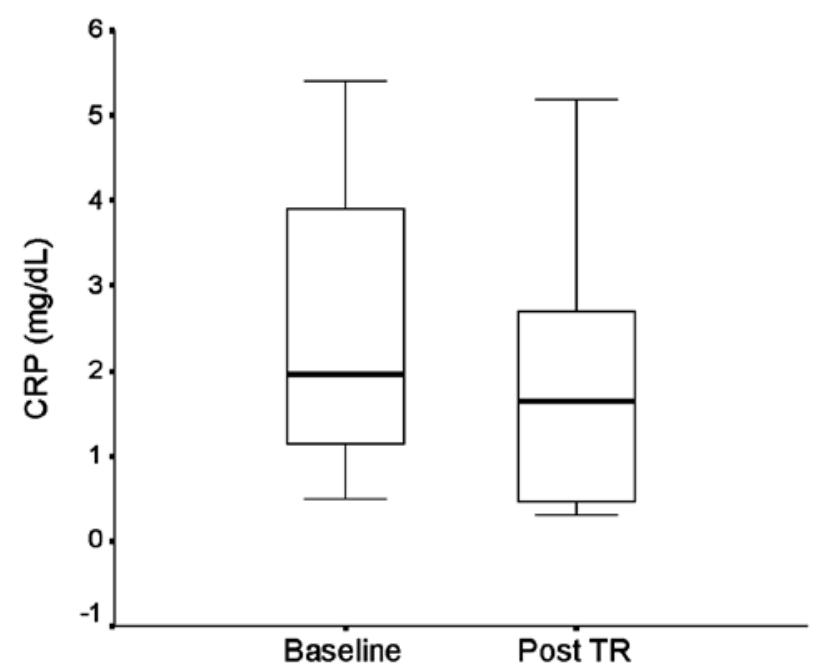

Figure 2. The effects of a combined strength and aerobic exercise training program (Post TR) on $\mathrm{C}$ reactive protein (CRP; $p=0.017)$. Values are presented as median and the length of the boxes represents the interquartile range.

to $9.1(1.7) \min (\mathrm{p}=0.012)$, while a $32.4 \%(\mathrm{p}=0.017)$ and $48.9 \%(p=0.014)$ increase was evident in the strength of upper and lower extremities, respectively.

\section{DISCUSSION}

The present study provides additional evidence 
Table 1. The effects of a systematic exercise training program on anthropometric, biochemical and physical fitness parameters

\begin{tabular}{lccc}
\hline & Baseline & Post TR & p value \\
\hline BW $(\mathrm{kg})$ & $81.3(23.4)$ & $79.6(23.7)$ & 0.007 \\
BMI $\left(\mathrm{kg} / \mathrm{m}^{2}\right)$ & $32.3(8.7)$ & $31.9(8.6)$ & 0.007 \\
Waist circumference & $106.2(27.0)$ & $102.1(24.4)$ & 0.005 \\
$(\mathrm{~cm})$ & & & \\
$\mathrm{HbA}_{1 \mathrm{c}}(\%)$ & $7.2(1.5)$ & $6.7(0.8)$ & 0.016 \\
HOMA-IR & $3.0(3.0)$ & $3.3(3.1)$ & 0.508 \\
IL-6 $(\mathrm{pg} / \mathrm{mL})$ & $6.1(3.3)$ & $6.1(2.6)$ & 0.263 \\
IL-10 $(\mathrm{pg} / \mathrm{mL})$ & $3.7(1.5)$ & $3.4(1.4)$ & 0.484 \\
INF- $\gamma(\mathrm{pg} / \mathrm{mL})$ & $15.7(16.0)$ & $14.5(17.2)$ & 0.980 \\
TNF- $\alpha(\mathrm{pg} / \mathrm{mL})$ & $2.2(1.7)$ & $2.3(1.5)$ & 0.326 \\
Stress test duration $(\mathrm{min})$ & $7.2(2.5)$ & $9.1(1.7)$ & 0.012 \\
Bench press strength $(\mathrm{kg})$ & $25.0(7.5)$ & $35.0(5.0)$ & 0.017 \\
Knee extension & $22.5(5.0)$ & $35.0(7.5)$ & 0.014 \\
strength $(\mathrm{kg})$ & & & \\
\hline
\end{tabular}

Values are presented as median (interquartile range). The baseline values were compared with the post training values within groups via the paired samples Wilcoxon test ( $p=0.05$ significant). Post TR, Post training; BW, body weight; BMI, body mass index; $\mathrm{HbA}_{1 c}$, glycated hemoglobin; HOMA-IR, homeostasis model of insulin resistance; TNF- $\alpha$, tumor necrosis factor- $\alpha$; INF- $\gamma$, interferon- $\gamma$; IL-6, interleukin-6; IL-10, interleukin-10.

of a rise in serum TGF- $\beta 1$ after systematic exercise in patients with DM2, whereas an anti-inflammatory protective effect is exerted by a decrease in serum hs-CRP levels and the stabilization of cytokines. An increase of serum TGF- $\beta 1$ levels has also been noted after strength training in healthy adults ${ }^{26}$ and in patients with DM2. ${ }^{27}$

In agreement with other studies, ${ }^{16,17,29-31}$ exercise training in our patients induced favourable adaptations in anthropometric characteristics, glucose control, physical fitness and muscle strength (Table 1). The increase of serum TGF- $\beta 1$, which functions as an anti-inflammatory growth factor and has an impact on plaque stability, ${ }^{6}$ is of additional clinical importance.

Indeed, exercise stimulus may act as the mechanical strain required to increase TGF- $\beta 1$ mRNA expression and this effect is strain dependent. ${ }^{11}$ Few studies have examined the effects of exercise on TGF- $\beta 1$. Hering et $\mathrm{al}^{26}$ detected an increase in serum levels of TGF- $\beta 1$ after four weeks of strength training in healthy adults. Gordon et $\mathrm{al}^{27}$ showed increased TGF- $\beta 1$ transcript in skeletal muscle of patients with DM2 after strength training. Our study confirms the data obtained from muscle biopsies using the more feasible procedure of blood sampling in patients with DM2. Therefore, the increased levels of serum TGF- $\beta 1$ after exercise achieved in our patients may indicate a protective effect leading to prevention or reduction of atherosclerosis progression. ${ }^{8}$

Elevated CRP concentration is considered a marker of subclinical inflammation and is associated with an increased risk of developing cardiovascular disease. ${ }^{4,32}$ A number of studies indicated that exercise stress has a protective cardiovascular and anti-inflammatory effect. ${ }^{23,24,33}$ In accordance with the aforementioned studies, ${ }^{23,24,33}$ we observed that eight weeks of combined exercise training was associated with significantly reduced levels of hs-CRP, but without alterations in IL-6, IL-10, INF- $\gamma$ and TNF- $\alpha$ in patients with DM2. It seems that the favourable effect of regular exercise was associated with CRP reduction and stabilization of cytokines, which is in line with other studies. ${ }^{23,33}$ Probably longer period is required for an effect on ILs, INF- $\gamma$ and TNF- $\alpha$. Indeed, Balducci et al, ${ }^{34}$ in a study of patients with DM2, demonstrated a decrease in hs-CRP and IL-6 and an increase in the anti-inflammatory cytokines (IL-4 and IL-10) after twelve months of exercise training, whereas no significant changes were found in the first three months of training, thus indicating differences in the exercise adaptation of these inflammation related cytokines. Furthermore, other factors which significantly change with exercise training are stronger determinants of CRP concentrations than of other cytokines.

It is well known that an intervention program induces favourable adaptations in the metabolic and inflammatory profile of patients with DM2. Either aerobic or resistance exercise training alone control the glucose metabolism in patients with DM2, but the results are better with a combined aerobic and resistance training regimen. ${ }^{18}$ In addition, Balducci et $\mathrm{al}^{34}$ observed a decrease in hs-CRP and IL- 6 and an increase in IL-4 and IL-10 in patients with DM2. The changes were more pronounced in the exercise training regimen which combines aerobic and strength training, compared to aerobic or strength training alone. 
Exercise induces vascular shear stress and has been shown to increase angiogenic growth factors. ${ }^{35} \mathrm{TGF}-\beta 1$ and vascular endothelial growth factor (VEGF) are consistently considered as growth factors mediating angiogenesis ${ }^{36}$ and regeneration. ${ }^{37}$ It is not known, however, to what degree exercise is capable of modifying the diabetes induced alterations in the vascular bed. The measurement of VEGF could possibly help in elucidating exercise induced alterations in circulating biomarkers.

Defining the appropriate duration, volume and intensity of exercise training in patients with DM2 required for the achievement of optimal levels of TGF- $\beta 1$, hs-CRP and cytokines, could possibly assure a more atheroprotective milieu. With the training program used in our study a $50.4 \%$ increase in TGF- $\beta 1$ and a $24.1 \%$ reduction in CRP, was achieved. These changes may well be associated with improved vascular tissue responsiveness. The findings might also support the potential importance of these factors for the prevention and treatment of vascular disease in diabetes.

The effect of exercise on physical fitness and on well recognized risk factors of cardiovascular disease is established; however, TGF- $\beta 1$ could be one more factor accounting for the link between systemic inflammation and diabetes complications, especially atherosclerosis. The latter represents the main cause of morbidity and mortality in patients with DM2. One could suggest that the increase in TGF- $\beta 1$ levels with exercise recruits a possible mechanism protecting from cardiovascular morbidity in patients with DM2. Up-regulating TGF- $\beta 1$ activity and increasing TGF levels would lead to prevention or reduction of atherosclerotic plaque production in diabetic individuals as well.

The main limitation of our study is the lack of a control group. It however furnishes novel information on the importance of the anti-atherogenic and anti-inflammatory effects of the exercise program applied. In fact, our intention was to maintain the homogeneity in our participants, which is difficult to achieve in a control group of patients with diabetes mellitus.

\section{CONCLUSION}

The present data suggest that a systematic training regimen which combines strength and aerobic exercise induces anti-inflammatory effects. The suppressed inflammation is reflected in the decreased levels of hs-CRP and the increased levels of TGF- $\beta 1$. Thus, systematic exercise should be considered in the treatment of DM2 not only for a better management of hyperglycemia, body composition and physical fitness but also for its anti-atherogenic and anti-inflammatory benefits.

\section{No conflict of interest declared}

This study was funded by the EPEAEK project

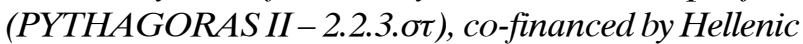
(25\%) and European Community (75\%) funds

\section{REFERENCES}

1. Spranger J, Kroke A, Möhlig M, et al, 2003 Inflammatory cytokines and the risk to develop type 2 diabetes: results of the prospective population-based European Prospective Investigation into Cancer and Nutrition (EPIC)-Potsdam Study. Diabetes 52: 812-817.

2. Dandona P, Aljada A, Bandyopadhyay A, 2004 Inflammation: the link between insulin resistance, obesity and diabetes. Trends Immunol 25: 4-7.

3. Libby P, Ridker PM, Maseri A, 2002 Inflammation and atherosclerosis. Circulation 105: 1135-1143.

4. Ridker PM, Rifai N, Rose L, Buring JE, Cook NR, 2002 Comparison of C-reactive protein and low-density lipoprotein cholesterol levels in the prediction of first cardiovascular events. N Engl J Med 347: 1557-1565.

5. Ross R, 1999 Atherosclerosis - an inflammatory disease. N Engl J Med 340: 115-126.

6. Feinberg MW, Jain MK, Werner F, et al, 2000 Transforming growth factor-beta 1 inhibits cytokine-mediated induction of human metalloelastase in macrophages. J Biol Chem 275: 25766-25773.

7. Lutgens E, Gijbels M, Smook M, et al, 2002 Transforming Growth Factor- $\beta$ mediates balance between inflammation and fibrosis during plaque progression. Arterioscler Thromb Vasc Biol 22: 975-982.

8. Grainger DJ, 2004 Transforming growth factor beta and atherosclerosis: so far, so good for the protective cytokine hypothesis. Arterioscler Thromb Vasc Biol 24: 399-404.

9. Mallat Z, Gojova A, Marchiol-Fournigault C, et al, 2001 Inhibition of transforming growth factor- $\beta 1$ signalling accelerates atherosclerosis and induces an unstable plaque phenotype in mice. Circ Res 89: 930-934. 
10. Tashiro H, Shimokawa H, Sadamatu K, Yamamoto K, 2002 Prognostic significance of plasma concentrations of transforming growth factor- $\beta$ in patients with coronary artery disease. Coron Artery Dis 13: 139-143.

11. O'Callaghan CJ, Williams B, 2000 Mechanical StrainInduced Extracellular Matrix Production by Human Vascular Smooth Muscle Cells Role of TGF-b1. Hypertension 36: 319-324.

12. Boulé NG, Haddad E, Kenny GP, Wells GA, Sigal RJ, 2001 Effects of exercise on glycemic control and body mass in type 2 diabetes mellitus: a meta-analysis of controlled clinical trials. JAMA 286: 1218-1227.

13. Dela F, 2007 Other adaptations to training/inactivity in type 2 diabetics and other groups with insulin resistance: emphasis on prevention of CHD. Appl Physiol Nutr Metab 32: 602-606.

14. Stewart KJ, 2002 Exercise training and the cardiovascular consequences of type 2 diabetes and hypertension: plausible mechanisms for improving cardiovascular health. JAMA 288: 1622-1631.

15. Colberg SR, Sigal RJ, Fernhall B, et al, 2010 Exercise and type 2 diabetes: the American College of Sports Medicine and the American Diabetes Association: joint position statement. Diabetes Care 33: 147-167.

16. Boulé NG, Weisnagel SJ, Lakka TA, et al, 2005 Effects of exercise training on glucose homeostasis. The HERITAGE family study. Diabetes Care 28: 120-126.

17. Cuff DJ, Meneilly GS, Martin A, et al, 2003 Effective exercise modality to reduce insulin resistance in women with type 2 diabetes. Diabetes Care 26: 2977-2982.

18. Sigal RJ, Kenny GP, Boulé NG, et al, 2007 Effects of aerobic training, resistance training, or both on glycemic control in type 2 diabetes: a randomized trial. Ann Intern Med 147: 357-369.

19. Sigal RJ, Kenny GP, Wasserman DH, Castaneda-Sceppa C, White RD, 2006 Physical activity/exercise and type 2 diabetes: a consensus statement from the American Diabetes Association. Diabetes Care 29: 1433-1438.

20. Tokmakidis SP, Zois CE, Volaklis KA, Kotsa K, Touvra AM, 2004 The effects of a combined strength and aerobic exercise program on glucose control and insulin action in women with type 2 diabetes. Eur J Appl Physiol 92: 437-442.

21. Zois CE, Tokmakidis SP, Volaklis KA, et al, 2009 Lipoprotein profile, glycemic control and physical fitness after strength and aerobic training in post-menopausal women with type 2 diabetes. Eur J Appl Physiol 106: 901-907.

22. Pedersen BK, 2006 The anti-inflammatory effect of exercise: its role in diabetes and cardiovascular disease control. Essays Biochem 42: 105-117.

23. Giannopoulou I, Fernhall B, Carhart R, et al, 2005 Effects of diet and/or exercise on the adipocytokine and inflammatory cytokine levels of postmenopausal women with type 2 diabetes. Metabolism 54: 866-875.

24. Oberbach A, Tönjes A, Klöting N, et al, 2006 Effect of a
4 week physical training program on plasma concentrations of inflammatory markers in patients with abnormal glucose tolerance. Eur J Endocrinol 154: 577-585.

25. Zoppini G, Targher G, Zamboni C, et al, 2006 Effects of moderate-intensity exercise training on plasma biomarkers of inflammation and endothelial dysfunction in older patients with type 2 diabetes. Nutr Metab Cardiovasc Dis 16: 543-549.

26. Hering S, Jost C, Schulz H, Hellmich B, Schatz H, Pfeiffer AFH, 2002 Circulating transforming growth factor- $\beta 1$ (TGF - $\beta 1$ ) is elevated in extensive exercise. Eur J Appl Physiol 86: 406-410.

27. Gordon PL, Vannier E, Hamada K, et al, 2006 Resistance training alters cytokine gene expression in skeletal muscle of adults with type 2 diabetes. Int J Immunopathol Pharmacol 19: 739-749.

28. Matthews DR, Hosker JP, Rudenski AS, et al, 1985 Homeostasis model assessment: insulin resistance and beta-cell function from fasting plasma glucose and insulin concentrations in man. Diabetologia 28: 412-419.

29. Boulé NG, Kenny GP, Haddad E, Wells GA, Sigal RJ, 2003 Meta-analysis of the effect of structured exercise training on cardiorespiratory fitness in Type 2 diabetes mellitus. Diabetologia 46: 1071-1081.

30. Church TS, Cheng YJ, Earnest CP, et al, 2004 Exercise capacity and body composition as predictors of mortality among men with diabetes. Diabetes Care 27: 83-88.

31. Cauza E, Hanusch-Enserer U, Strasser B, et al, 2005 The relative benefits of endurance and strength training on the metabolic factors and muscle function of people with type 2 diabetes mellitus. Arch Phys Med Rehabil 86: $1527-1533$.

32. Jager A, van Hinsbergh VW, Kostense PJ, et al, 1999 von Willebrand factor, C-reactive protein, and 5-year mortality in diabetic and nondiabetic subjects: the Hoorn Study. Arterioscler Thromb Vasc Biol 19: 3071-3078.

33. Dvoráková-Lorenzová A, Suchánek P, Havel PJ, et al, 2006 The decrease in C-reactive protein concentration after diet and physical activity induced weight reduction is associated with changes in plasma lipids, but not interleukin-6 or adiponectin. Metabolism 55: 359-365.

34. Balducci S, Zanuso S, Nicolucci A, et al, 2010 Antiinflammatory effect of exercise training in subjects with type 2 diabetes and the metabolic syndrome is dependent on exercise modalities and independent of weight loss. Nutr Metab Cardiovasc Dis 20: 608-617.

35. Danzig V, Míková B, Kuchynka P, et al, 2010 Levels of circulating biomarkers at rest and after exercise in coronary artery disease patients. Physiol Res 59: 385-392.

36. Tammela T, Enholm B, Alitalo K, Paavonen K, 2005 The biology of vascular endothelial growth factors. Cardiovasc Res 65: 550-563.

37. Rafii S, Lyden D, 2003 Therapeutic stem and progenitor cell transplantation for organ vascularization and regeneration. Nat Med 9: 702-712. 